\title{
Variabilidad intramétodo en medidas del flujo del acceso vascular con termodilución: ¿Un enemigo en los programas de seguimiento?
}

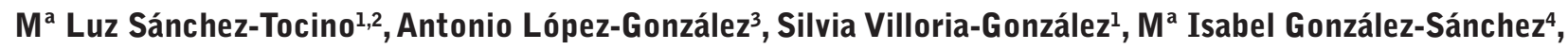 \\ Ana Silvia Puente-González ${ }^{5}$ \\ ${ }^{1}$ Centro de Hemodiálisis "Las Encinas". Fundación Renal Íñigo Álvarez de Toledo (FRIAT). Salamanca. España \\ ${ }^{2}$ Centro de Hemodiálisis "El Castañar". Fundación Renal Íñigo Álvarez de Toledo (FRIAT). Salamanca. España \\ ${ }^{3}$ Servicio de Nefrología. Hospital Universitario. A Coruña. España \\ ${ }^{4}$ Centro de Hemodiálisis "Los Llanos". Fundación Renal Íñigo Álvarez de Toledo (FRIAT). Madrid. España \\ ${ }^{5}$ Escuela Universitaria de Enfermería y Fisioterapia. Universidad de Salamanca. Salamanca. España
}

Como citar este artículo:

Sánchez-Tocino ML, López-González A, Villoria-González S, González-Sánchez MI, Puente-González AS. Variabilidad intramétodo en medidas del flujo del acceso vascular con termodilución: ¿Un enemigo en los programas de seguimiento?

Enferm Nefrol. 2020 Oct-Dic;23(4):353-360

\section{Resumen}

Objetivo: Analizar el impacto de la variabilidad intramétodo de la Termodilución (TD) en las medidas prospectivas de flujo de acceso $\left(Q_{A}\right)$ y su relación con los parámetros de seguimiento de primera generación.

Método: Estudio prospectivo. Se realizaron 2 mediciones consecutivas de $Q_{A}$ (M1 y M2) y un seguimiento (M3) en 6 meses. Se recogieron datos demográficos y parámetros de seguimiento de primera generación.

Resultado: Se analizaron 112 fistulas arteriovenosas(FAV). La mediana de la variabilidad generada entre M1 y M2 no difiere del porcentaje de variación de $Q_{A}$ a los 6 meses $(p=0,123)$. En el 16,8\% (14) de los pacientes el $Q_{A}$ ha disminuido más del $25 \%$ y en un $28,9 \%$ (24) aumentó más del 25\%. Se evidenció una ligera tendencia a aumentar el porcentaje de descenso de $Q_{A}$ a medida que el flujo de las fístulas es mayor $(r=-0,229$; $p=0,006$ ). Por otra parte, un descenso de $Q_{A}$ superior al $25 \%$ no se asoció a menor dosis de diálisis $(p=0,183)$, ni ha aumento significativo de la presión venosa dinámica $(p=0,823)$ ni al aumento de incidencias durante la punción $(p=0,823)$.

\section{Correspondencia: \\ $M^{\mathrm{a}}$ Luz Sánchez Tocino \\ Email:Isanchez@friat.es}

Conclusiones: La presencia de pacientes con aumento de flujo superior a la variabilidad intramétodo y la no asociación entre un descenso superior al $25 \%$ y cambios en otros parámetros de seguimiento, hace sospechar la presencia de errores de medición de QA. Frente a ello es conveniente el uso combinado con métodos de primera generación, tanto para establecer el QA basal como para interpretar los descensos en el seguimiento.

PALABRAS CLAVE: acceso vascular; flujo de acceso; termodilución; variabilidad intramétodo; seguimiento.

\section{Intra-method variability in the measurements of vascular access using the thermodilution technique: an enemy in follow-up programs?}

\begin{abstract}
Objective: To analyze the impact of the intra-method variability of thermodilution (TD) in the prospective measurements of the access flow $\left(Q_{A}\right)$ and the relationship with the first-generation monitoring parameters.
\end{abstract}

Method: Prospective study. Two consecutive $Q_{A}$ measurements ( $\mathrm{Ml}$ and $\mathrm{M} 2$ ) and a 6-month follow-up 
(M3) were performed. Demographic data and firstgeneration follow-up parameters were collected.

Result: 112 arteriovenous fistulas (AVF) were analyzed. The median variability generated between $M 1$ and M2 does not differ from the percentage of $Q_{A}$ variation at 6 months ( $p=0.123)$. In $16.8 \%$ (14) of the patients the QA has decreased by more than $25 \%$ and in $28.9 \%$ (24) it increased by more than $25 \%$. A slight tendency to increase the percentage of decrease in $Q_{A}$ when the fistula flow was higher was evidenced $(r=-0.229 ; p=0.006)$. On the other hand, a decrease in QA greater than $25 \%$ was not associated with a lower dose of dialysis $(p=0.183)$, nor did it have a significant increase in dynamic venous pressure $(p=0.823)$ or an increase in incidences during puncture $(p=0.823)$.

Conclusions: The presence of patients with an increase in flow greater than the intra-method variability, and the non-association between a decrease greater than $25 \%$ and changes in other follow-up parameters, raises suspicions about the presence of $Q_{A}$ measurement errors. In relation to this, the combined use with firstgeneration methods is convenient, both to establish the baseline $Q_{A}$ and to interpret the decreases in follow-up.

KEYWORDS: vascular access; access flow; thermodilution; intra-method variability, monitoring.

\section{Introducción}

El acceso vascular (AV) en el paciente de hemodiálisis supone una parte fundamental de su tratamiento, de la cual dependerá la calidad de diálisis y su morbi-mortalidad a largo plazo․ La fistula arteriovenosa (FAV) nativa seguida del injerto tienen menor tasa de complicaciones frente al catéter ${ }^{2,3}$. Según recomienda la Guía Clínica Española del Acceso Vascular para Hemodiáli$\operatorname{sis}^{4} y$, a pesar del cambio en el perfil del paciente que inicia hemodiálisis ${ }^{5}$, se intenta detener el aumento de la prevalencia de catéteres mediante la aplicación de programas de monitorización y vigilancia estrecha del $\mathrm{AV}$, que permitirá el diagnostico de las estenosis cuyo tratamiento temprano podría evitar la trombosis ${ }^{6}$.

Uno de los métodos de cribado o screening que forman parte de los programas de vigilancia de la FAV son los llamados de segunda generación, que nos permiten calcular de forma no invasiva el flujo sanguíneo del acceso $\left(Q_{A}\right)^{2}$. Entre ellos destacan los métodos dilucionales que, desde su introducción en el año 1995, han supuesto un cambio cualitativo en el estudio de la FAV ya que, por primera vez, era posible efectuar la estimación no invasiva de $Q_{A}{ }^{7}$. Desde entonces se han descrito diversas técnicas dilucionales que permiten determinar el $Q_{A}$ durante la hemodiálisis (HD) no existiendo una preferencia clara por ninguno de ellos por su alto grado de concordancia ${ }^{8,9}$. En la actualidad, uno de los más usados es la Termodilución (TD) puesto que ofrece como ventaja una mayor funcionalidad al tratarse de un biosensor integrado en el monitor.

En la interpretación de las medidas del QA, su evolución temporal tiene un valor diagnóstico superior a la hora de detectar estenosis de la FAV que determinaciones aisladas. En este sentido, una disminución temporal de QA mayor al $20-25 \%$ en relación con la medición basal debería ser criterio suficiente para el control de ese AV por otro método directo ${ }^{4}$. Sin embargo, diversos autores consideran que estos porcentajes de descenso de $Q_{A}$ pueden ser secundarios a otros factores. Paulson y cols. los asocian a cambios hemodinámicos, los cuales pueden llegar a alcanzar hasta el $33 \%{ }^{9}$. Por otro lado, Schneditz et.al en su estudio de validación de la TD para la determinación del $Q_{A}$, describe la presencia de una variabilidad intramétodo que, sin tener en cuenta cambios hemodinámicos, puede generar un descenso del $Q_{A}$ de hasta un $23 \%{ }^{11}$.

Ante estas discrepancias en la interpretación diagnóstica del $Q_{A}$, el papel de los métodos de primera generación en el seguimiento de la $\mathrm{FAV}$ se ha convertido en indiscutible. A pesar de que perdieron cierto protagonismo con el auge de los métodos dilucionales y la difusión de la EcoDoppler, la monitorización de parámetros como las presiones dinámicas y el índice de eficacia dialítica (KT) así como las incidencias durante la punción del acceso nos aportan datos fundamentales para un diagnóstico precoz de la estenosis ${ }^{4}$.

Por lo tanto, con el fin de mejorar la eficacia de los programas de seguimiento y optimizar los recursos al evitar la sobresaturación de los servicios de radiología y cirugía vascular con casos innecesarios, es de vital importancia conocer las limitaciones o posibles sesgos asociados a la utilización de las técnicas dilucionales que calculan el $Q_{A}$, así como, las estrategias para minimizarlos. Para ello, el objetivo principal de este estudio fué analizar el impacto de la variabilidad intramétodo de la TD en las medidas prospectivas de QA y su relación con los parámetros de seguimiento de primera generación. El objetivo secundario fué analizar las incidencias 
ocurridas en el periodo de estudio y su relación con las medidas de $Q$ A.

\section{Material y Método}

Se llevó a cabo un estudio observacional prospectivo. Se estudiaron las FAV pertenecientes a paciente en programa de hemodiálisis periódica en tres centros periféricos de Castilla y León y Madrid pertenecientes a la Fundación Renal Iñigo Álvarez de Toledo. Todos los pacientes fueron informados y dieron su consentimiento. Se definió como criterio de inclusión, pacientes cuya FAV contara con un periodo de maduración superior a un mes. Se excluyeron los pacientes a los que no se pudo determinar el flujo de acceso mediante métodos dilucionales (retorno sanguíneo en vena distinta a la arterializada, presencia de vasos colaterales por los que puede perderse el indicador, FAV en las que se ha detectado una alta recirculación del propio acceso).

Como variable primaria, se registró el $Q_{A}$ mediante TD. Para obtención las medidas, se utilizó el sensor de temperatura sanguínea BTM $^{\circledR}$ (Blood Temperature Monitor) incorporado en los monitores de hemodiálisis 5008 de Fresenius que permite calcular el porcentaje de recirculación existente con las líneas de hemodiálisis en configuración normal e invertida. A partir de ambos valores de recirculación, el QA se calcula mediante la siguiente ecuación ${ }^{12}$ :

$$
Q_{A}=\frac{\left(Q_{S}-T U F\right) \times\left[1-R_{x}-R_{N}+R_{x} \times R_{N}\right]}{R_{X}-R_{X} \times R_{N}-\left(\frac{Q_{S}-T U F}{Q_{S}}\right) \times\left(R_{N}-R_{X} \times R_{N}\right)}
$$

Donde QS es el flujo efectivo de sangre ( $\mathrm{ml} / \mathrm{min})$; TUF es la tasa de ultrafiltración ( $\mathrm{ml} / \mathrm{min})$; RN es la recirculación obtenida con las líneas de hemodiálisis en posición normal y RX es la recirculación obtenida con las líneas de hemodiálisis en posición invertida.

Las medidas se realizaron utilizando el método descrito por el fabricante. Este consiste en utilizar para el cálculo matemático, el valor medio de dos medidas de RN y una única medida de Rx obtenidos en este mismo orden. Se llevaron a cabo dos medidas basales en sesiones consecutivas ( $M 1$ y M2) y una tercera medida de seguimiento trascurridos seis meses aproximadamente (M3). Para el análisis del porcentaje de descenso en las medidas de seguimiento se tomó como flujo basal la media entre M1 y M2.
Todas las determinaciones se realizaron en los primeros 90 minutos de tratamiento y se mantuvo una ratio de ultrafiltración constante para evitar influencias en la medida del QA producidas por los cambios de volemia4. Además, se utilizó un flujo de bomba de $250 \mathrm{ml} / \mathrm{min}$, la distancia entre agujas fue la misma en cada proceso y las punciones fueron en posición anterógrada y con el bisel hacia abajo, según las últimas recomendaciones ${ }^{12}$.

También, se recopiló la tensión arterial media (PAM) en los distintos momentos de medición. Se recogió datos en la medida basal y la medida de seguimiento referentes a la eficacia dialítica determinada por dialisancia iónica $(\mathrm{Kt}$ ) y presión venosa (PV) para determinar la proporción de pacientes cuyo Kt decreció más de $10 \%$ y cuya PV aumentó más de un $10 \%$, sin que existieran variaciones del flujo de bomba mayor del $10 \%$. Como complemento, se obtuvieron variables demográficas (edad y tiempo en hemodiálisis) y relacionadas con la FAV (tipo acceso vascular, localización y tiempo del mismo).

Todos los parámetros del estudio se recogieron a través de la historia clínica informática mediante el programa Nefrosoft ${ }^{\circledR}$ V.6.7.4.

\section{Análisis estadístico}

El abordaje estadístico se llevó a cabo con el programa IBM SPSS Statistics v20. Las variables cuantitativas se presentaron como media y desviación estándar o mediana y rango intercuartílico, atendiendo al criterio de normalidad determinado a través de la prueba de Kolmogorov-Smirnov. Se concretó el análisis de significación estadística para $p \leq 0,05$ utilizando la prueba t-Student o Wilcoxon según procedió. Para el análisis de correlación se utilizó el coeficiente de Spearman. Para el estudio de la concordancia se utilizó el coeficiente de correlación intraclase (ICC) previa transformación logarítmica de los datos para ajustarse al criterio de normalidad y el método Bland-Altman ${ }^{13}$. Este método consiste en la representación gráfica de las diferencias de entre M1 y M2 frente al promedio de ambas medidas, mostrando los límites de acuerdo al $95 \%$ (percentil 2,5 y 97,5 ) y la variabilidad promedio a través de la mediana de las diferencias (variabilidad intramétodo). Por último, se utilizó el test de chi cuadrado para analizar la asociación entre la variación prospectiva de $Q_{A}$ indicativa de valoración del AV por prueba de imagen y los métodos de primera generación de seguimiento de la FAV. 


\section{Resultados}

Se han analizado 112 fístulas arteriovenosas pertenecen a pacientes cuya media de edad fue $68,5 \pm 13,6$ y mediana de tiempo de permanencia en hemodiálisis de 36,2 (36,2 $-112,8$ ) meses. El 58,9\% (66) de las fístulas eran radiales y el $41,1 \%$ (46) restantes humerales. La mediana del tiempo del AV fue de $45(25,7-71,8)$ meses.

La mediana de $Q_{A}$ en $\mathrm{Ml}$ fue de 754,6 $\mathrm{ml} / \mathrm{min}$ (rango 472,4$1151,4 \mathrm{ml} / \mathrm{min}$ ) frente a $\mathrm{M} 2$ que fue de $761,2 \mathrm{ml} / \mathrm{min}$ (rango $505,2-1164,2 \mathrm{ml} / \mathrm{min})$; con un ICC de 0,850 (IC:95\%: 0,7900,$895 ; p=0,910$. Análisis de correlación $r=0,851 ; p=0,000$ ). No se evidenció condicionamiento de la PAM $(p=0,634)$ en dichos análisis cuyos valores registrados en los distintos momentos de medición fueron $88,7+14,1 \mathrm{mmHg}$ en $\mathrm{M} 1$ y $88,6+13,7 \mathrm{mmHg}$ en M2.

En la Figura 1, a través del procedimiento de Bland Alman, se puede apreciar que a medida que se incrementa la magnitud del $Q_{A}$ la variabilidad entre medidas aumenta. En este mismo gráfico se denota una extensa amplitud de los límites de acuerdo observados. Esto puede provocar errores de interpretación de los mismos, ya que, a magnitudes de flujo bajas, su impacto será mayor. Ante esto, resulta útil expresar la variabilidad intramétodo y los límites de acuerdo al 95\% como porcentaje.

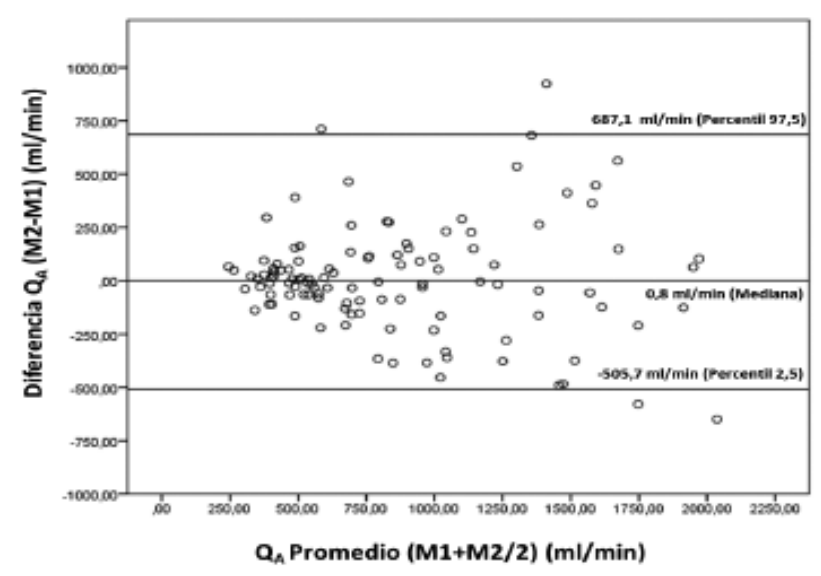

Figura 1. Gráfico de Bland-Altman.
Tabla 1. Descripción de pacientes con intervención terapéutica.

\begin{tabular}{|c|c|c|c|c|c|}
\hline $\begin{array}{c}\text { Caso } \\
(\mathrm{ml} / \mathrm{min})\end{array}$ & QA Basal* & Signos Primarios & Confirmación & Reparación & $\begin{array}{l}\text { QA Seguimiento } \\
(\mathrm{ml} / \mathrm{min})\end{array}$ \\
\hline 1 & 339 & Descenso KT & Ecografía & Angioplastia & 530 \\
\hline 2 & 305 & No & Ecografía & No Necesaria & 552 \\
\hline 3 & 263 & Descenso KT & Fistulografía & Angioplastia & 582 \\
\hline 4 & 245 & $\begin{array}{c}\text { Elevación PV } \\
\text { Dificultad Punción }\end{array}$ & Fistulografía & Angioplastia & 451 \\
\hline 5 & 375 & $\begin{array}{c}\text { Elevación PV } \\
\text { Dificultad Punción }\end{array}$ & Fistulografía & Angioplastia & 786 \\
\hline 6 & 687 & $\begin{array}{c}\text { Elevación PV } \\
\text { Dificultad Punción } \\
\text { Aumento Hemostasia }\end{array}$ & Fistulografía & Angioplastia & 671 \\
\hline 7 & 695 & Descenso KT & Fistulografía & Angioplastia & 1790 \\
\hline 8 & 848 & $\begin{array}{c}\text { Elevación PV } \\
\text { Dificultad Punción }\end{array}$ & Fistulografía & Angioplastia & 1026 \\
\hline 9 & 837 & Dificultad Punción & Fistulografía & Trombectomía & 738 \\
\hline 10 & 537 & Trombosis & - & CVC & - \\
\hline
\end{tabular}

Así, el valor mediano de la variabilidad generada entre M1 y R2 fue 1,4\% (IC95\%: -34\%,126\%).

Para el análisis de las medidas de seguimiento se han incluido a estudio el $74,1 \%$ (83) de los pacientes. En la Tabla 1 se describe la intervención terapéutica realizada en los AV durante el período de estudio. En la Tabla 2 se muestran los datos de las variables estudiadas obtenidos en la medición prospectiva a los 6 meses frente a sus valores basales de los 83 pacientes incluidos finalmente.

Tabla 2. Valores medios y desviación típica de las variables estudiadas obtenidos en la medición prospectiva a los 6 meses frente a sus valores basales $(n=83)$.

\begin{tabular}{|l|c|c|c|}
\hline & $\begin{array}{c}\text { Medida } \\
\text { Basal* }\end{array}$ & $\begin{array}{c}\text { Medida } \\
\text { prospectiva }\end{array}$ & p \\
\hline Flujo Acceso (ml/min) & $741,5 \pm 435,1$ & $973 \pm 511,6$ & 0,168 \\
\hline PAM (mmHg) & $91,9+14,6$ & $90,49+14$ & 0,334 \\
\hline Flujo de Bomba (ml/min) & $371,9+32,5$ & $378,69+30,3$ & 0,005 \\
\hline Presión Venosa (mmHg) & $161,6+20,1$ & $168,77+19,4$ & 0,000 \\
\hline KT (I) & $49,3+6,4$ & $50,04+6,5$ & 0,212 \\
\hline
\end{tabular}

*Valor medio de M1 y M2. 
La variación mediana de $Q_{A}$ a los 6 meses de seguimiento fue de $2,1(-16,7-36,1) \%$, valor que no difiere con respecto a la variabilidad intramétodo $(p=0,717)$. En el 44,6\% (37) de los pacientes el $Q_{A}$ ha disminuido y de todos ellos ese descenso fue superior al $25 \%$ en un $37,83 \%$ (14). En un 28,9\% (24) se evidenció un aumento de flujo mayor al $25 \%$.

En el dispersograma de la Figura 2 se muestra el porcentaje de variación del QA a los 6 meses frente al flujo basal. Se evidenció una ligera tendencia a aumentar el descenso de $Q_{A}$ a medida que el flujo de las fístulas es mayor. Atendiendo a esto, la mediana del QA basal de los pacientes cuya medición prospectiva decreció más del $25 \%$ fue $1184,69+517 \mathrm{ml} / \mathrm{min}$ frente a los $906,59+403,68 \mathrm{ml} / \mathrm{min}$ de los pacientes cuya variación no alcanzó dicho criterio $(p=0,028)$.

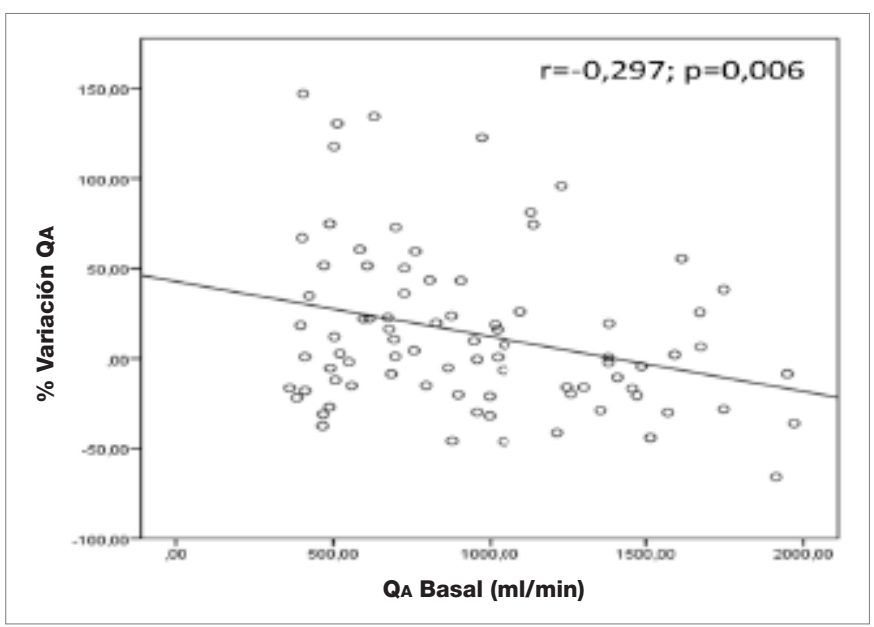

Figura 2. Porcentaje de Variación QA vs QA Basal.

Por otra parte, en la Tabla 3 se muestran los datos del análisis de asociación entre el descenso de $Q_{A}$ con la disminución de dosis de diálisis, el aumento significativo de la presión venosa dinámica y la incidencia durante la punción no evidenciándose significancia estadística.

Tabla 3. Análisis de la relación entre variables a estudio en pacientes SIN variación del flujo de bomba $\geq 10 \%$. $n=75$.

\begin{tabular}{|l|c|c|c|c|}
\hline & & $\begin{array}{c}\text { Descenso QA } \geq 25 \% \\
\%(n)\end{array}$ & $\begin{array}{c}\text { Descenso QA }<25 \% \\
\%(n)\end{array}$ & $p$ \\
\hline Disminución KT $\geq 10 \%$ & Si & $33,3 \%(3)$ & $66,7 \%(6)$ & 0,183 \\
& No & $15,2 \%(10)$ & $84,8 \%(56)$ & \\
\hline \multirow{2}{*}{ Aumento PV $\geq 15 \%$} & Si & $14,3 \%(1)$ & $85,7 \%(6)$ & 0,823 \\
& No & $17,6 \%(12)$ & $82,4 \%(56)$ & \\
\hline \multirow{2}{*}{3 o más Repunciones } & Si & $14,3 \%(1)$ & $85,7 \%(6)$ & 0,823 \\
& No & $17,6 \%(12)$ & $82,7 \%(62)$ & \\
\hline
\end{tabular}

\section{Discusión}

A pesar de lo fundamental que resulta una adecuada coordinación multidisciplinar que garantice la supervivencia del $\mathrm{AV}$, no todas las unidades diálisis disponen de un acceso directo y fácil a los servicios de cirugía vascular o radiología ${ }^{14}$. Esto, junto a la importancia del control del gasto sanitario, hace esencial la optimización del manejo de los métodos de vigilancia del $\mathrm{AV}$, como es la medida del $\mathrm{QA}_{\mathrm{A}}$ mediante el estudio de los posibles errores de medición que puedan generar impacto en la sensibilidad y especificidad de la prueba.

En nuestro estudio, con el análisis de dos determinaciones consecutivas de QA bajo las mismas condiciones de medición y sin evidenciarse cambios hemodinámicos significativos, se pone de manifiesto la diferencia entre medidas a pesar de los buenos resultados que reporta el análisis de concordancia. Por tanto, en nuestro estudio se evidencia la presencia de una variabilidad intramétodo cuyos valores siguen la línea de lo descrito en otros estudios ${ }^{12}$.

El comportamiento de dicha variabilidad, observado a través del procedimiento de Bland-Altman, no describe ningún patrón sistemático específico. Sin embargo, resulta evidente la muy buena concordancia de medidas para valores de $Q_{A}$ inferiores a $700 \mathrm{ml} / \mathrm{min}$, empeorando a medida que la magnitud de flujo aumenta. Dicho fenómeno ya fue descrito previamente por otros autores en sus estudios de concordancia sobre métodos dilucionales ${ }^{15,16}$, donde se le atribuyo la causa, a las condiciones de mezcla del indicador utilizado. En valores altos de $Q_{A}$, el perfil de flujo laminar se intensifica creándose las peores condiciones de mezcla posibles. Esto puede provocar que parte del indicador se escape sin ser detectado por el sensor de medición generando valores de $Q_{A}$ sobreestimados. Por otro lado, a pesar de que en nuestro estudio se ha controlado las variables distancia y dirección de las agujas y bisel, la colocación de la aguja dentro de la luz del vaso podría generar un aumento anormal de la turbulencia dando lugar por tanto a una infraestimación del flujo ${ }^{8}$.

Es incuestionable que el papel de la variabilidad intramétodo descrita adquiere especial relevancia en el análisis de las medidas de seguimiento. Según las recomendaciones de las principales guías clínicas, las FAV en 
las que el QA haya descendido un $20-25 \%$ deberían ser valoradas por pruebas de imagen ${ }^{4,5}$. En nuestro estudio, en el $45 \%$ de los pacientes el $Q_{A}$ ha disminuido, siendo el descenso superior al $25 \%$ en 14 pacientes. Sin embargo, ¿Este es criterio suficiente para derivarlos a revisión a través de medios de imagen? La respuesta a esta pregunta resulta compleja debido fundamentalmente a esta variabilidad intramétodo. Así, en 24 pacientes de nuestro estudio, a los que no se realizó intervención terapéutica, se evidenció un aumento de QA mayor del $25 \%$. Este hecho, nos hace pensar que al igual que interpretamos como errores de medida los aumentos espontáneos de $Q_{A}$, también puede haber errores en los pacientes con descenso de flujo en el seguimiento.

Por este motivo, creemos que en la interpretación prospectiva de la variación del QA cobra vital importancia la medición basal. En las FAV en las que el flujo disminuye hemos de plantearnos si partimos de una medida basal sobreestimada y para ello debemos ayudarnos de la información que nos aportan otros signos de primera generación ${ }^{17-19}$. En nuestro estudio a pesar de que el $25 \%$ pacientes tenían QA basal inferior a $500 \mathrm{ml} / \mathrm{min}$ (criterio suficiente para la realización de una prueba de imagen para el descarte de patología del $A V^{4,5}$ ) solo un $18 \%$ de estos se revisaron puesto que la mayoría se acompañaba de otros signos como descenso de Kt, dificultad de la punción o aumento de PV durante las sesiones previas a la medición, siendo necesaria la intervención por parte de cirugía vascular.

Por otro lado, en el período del estudio, hubo un $6 \%$ de los pacientes que a pesar de presentar un QA basal aceptable se sometieron a prueba de imagen, dado que mostraron con posterioridad alteración de otros signos primarios de vigilancia. Igualmente, todos requirieron intervención, verificándose la presencia de estenosis por fistulografía. Consideramos que en estos casos una determinación de seguimiento más tempra$\mathrm{na}$, a los tres meses en lugar de a los seis meses, tal y como recomienda la bibliografía ${ }^{20,21}$, hubiera marcado la tendencia de estos AV a la pérdida de flujo.

Además, en aquellas fístulas con las que partimos de flujos bajos, consideramos consecuente aumentar la frecuencia de las determinaciones y prestar una mayor atención a la aparición de otros signos que puedan indicarnos una disfunción. Puesto que, en flujos inferiores a $700 \mathrm{ml} / \mathrm{min}$, la concordancia entre métodos es muy buena, lo que presupone que el valor basal del que partimos tiene menos variabilidad y, por tanto, menor riesgo de cometer errores de interpretación.

En cuanto a las FAV con altos flujos, los datos reportados en nuestro estudio demuestran una ligera tendencia a que aumente su porcentaje de descenso de $Q_{A}$ en las medidas de seguimiento. Esto corrobora el mayor impacto del error de medida a magnitudes de flujo elevadas. Además, en nuestro análisis observamos que los descensos de $Q_{A}$ superior al $25 \%$ no se asociaron a menor dosis de diálisis, ni ha aumento significativo de la presión venosa dinámica, ni al aumento de incidencias durante la punción. Tampoco se evidenció diferencias en el porcentaje de variación de flujo en la medida de seguimiento en los pacientes con y sin la presencia de al menos uno de estos criterios. Por estos motivos y del mismo modo que en las determinaciones de QA ecográficas se consideran como positivo los descensos mayores del $25 \%$ siempre y cuando los flujos sean inferiores a los $1000 \mathrm{ml} / \mathrm{min}^{4}$, creemos que este criterio ha de considerarse también para el uso de los métodos dilucionales.

Por último, un factor a tener en cuenta a la hora de optimizar el proceso de obtención del QA es la PAM registrada en el momento de medición. Aunque en nuestro estudio no se han evidenciado diferencias significativas en los distintos momentos de medición, existe evidencia de su papel determinante en las variaciones de $Q_{A}$ hasta el punto de que un $10 \%$ de disminución de la PAM puede llegar a reducir un $8 \%$ el $Q_{A}$ de la FAV 22.

Por tanto, a partir de los resultados obtenidos, podemos concluir que la variabilidad intramétodo de las medidas es considerable e imprevisible, tanto sobreestimando como infraestimando el resultado. Esta variabilidad es más relevante a medida que aumenta el flujo del acceso. En el análisis prospectivo no es tan evidente el impacto de la magnitud de flujo en el porcentaje de variación, aun así, se observó una tendencia al descenso del QA en flujos más altos. La presencia de pacientes con aumento de flujo superior a la variabilidad intramétodo y la no asociación entre un descenso superior al $25 \%$ y cambios en otros parámetros de seguimiento, hace sospechar la presencia de errores de medición. Frente a ello es conveniente el uso combinado con métodos de primera generación, tanto para establecer el QA basal como para interpretar los descensos en el seguimiento, como se demuestra en el análisis de casos mostrados. 
Recepción: 22-07-20

Aceptación: 7-11-20

Publicación: 30-12-20

\section{Bibliografía}

1. Rodríguez Hernández JA, López Pedret J, Piera L. El acceso vascular en España: análisis de su distribución, morbilidad y sistemas de monitorización. Nefrología. 2001;21(1):45-51.

2. Konner K, Nonnast-Daniel B, Ritz E. The arteriovenous fistula. J Am Soc Nephrol 2003;14(6):166980.

3. Blanco I, Rodríguez MA, Sánchez C. Evaluación de complicaciones de la fístula arteriovenosa para hemodiálisis según la técnica de canalización. Enferm Nefrol. 2017;20(2):167-77.

4. Ibeas J, Roca-Tey R, Villespín J, Moreno T, Moñux G, Martí-Monrós A et al. Guía Clínica Española del Acceso Vascular para Hemodiálisis. Enferm Nefrol. 2018;21(Supl 1):S1-256.

5. National Kidney Foundation. KDOQI Clinical Practice Guidelines and Clinical Practice Recommendations for 2006 Updates: Haemodialysis Adequacy, Peritoneal Dialysis Adequacy and Vascular Access. Am J Kidney Dis 2006;48(1):1-322.

6. Arenas MD, Malek T, López-Collado M, Gil MT, Moledous A, Morales A et al. Operation to remove tunnelled venous catheters in a dialysis unit. Is it possible to reverse the trend in their growing use? Nefrología. 2009;29(4):318-26.

7. Ibeas López J. Monitorización del acceso vascular: ¿Quo vadis? NefroPlus. 2011;4:11-20.

8. Krivitski NM. Theory and validation of access flow measurement by dilution technique during hemodialysis. Kidney Int. 1995;48:244-50.

9. Badr B, Bories $P$, Marais R, Frat B, Seigneuric $B$, Longlune $N$ et al. Transonic, thermodilution,

or ionic dialysance to manage vascular access: which method is best? Hemodialysis International 2014;18:127-35.

10. Paulson WD, Moist L, Lok CE. Vascular access surveillance: an ongoing controversy. Kidney Int 2012;81:132-42.

11. Schneditz D, Wang E, Levin NW. Validation of haemodialysis recirculation and access blood flow measured by thermodilution. Nephrol Dial Transplant 1999;14:376-83.

12. Parisotto MT, Schoder VU, Miriunis $C$, Grassmann AH, Scatizzi LP, Kaufmann P et al. Cannulation technique influences arteriovenous fistula and graft survival. Kidney Int. 2014;86(4):790-7.

13. Bland JM, Altman DG. Statistical methods for assessing agreement between two methods of clinical measurement. Lancet. 1986;1:307-10.

14. Tordoir J, Canaud B, Haage P, Konner K, Basci A, Fouque D, et al. EBPG on Vascular Access. Nephrol Dial Transplant. 2007;22(2):88-117.

15. Sánchez ML, López A, Sánchez A, Villoria S, Turrión V, Gascón M, Marina Burgos M, Sánchez $H$. Efecto de la dirección de la aguja arterial en la medición del flujo del acceso vascular con métodos dilucionales. Enferm Nefrol. 2017;20(4): 305-15.

16. Merino JL, Teruel JL, Galeano C, Fernández Lucas $M, 0$ caña J, Rivera M et al. Blood flow determination in vascular access withionic dialysance. $\mathrm{Ne}$ frologia. 2006;26:481-5.

17. Tessitore $N$, Bedogna V, Melilli E, Millardi D, Mansueto G, Lipari G, et al. In Search of an Optimal Bedside Screening Program for Arteriovenous Fistula Stenosis. Clin J Am Soc Nephrol. 2011;6: 819-26.

18. Salman L, Beathard G. Interventional Nephrology: Physical Examination as a Tool for Surveillance for the Hemodialysis Arteriovenous Access. Clin J Am Soc Nephrol. 2013;8:1220-7.

19. Tessitore N, Bedogna V, Verlato G, Poli A. Clinical access assessment. J Vasc Access. 2014;15(7): 20-7. 
20. Tordoir J, Canaud B, Haage P, Konner K, Basci A, Fouque D, et al. EBPG on Vascular Access. Nephrol Dial Transplant. 2007;22(2):88-117.

21. Jindal K, Chan CT, Deziel C, Hirsch D, Soroka SD, Tonelli M, et al; Canadian Society of Nephrology Committee for Clinical Practice Guidelines. Hemodialysis clinical practice guidelines for the $\mathrm{Ca}$ nadian Society of Nephrology. J Am Soc Nephrol. 2006;17(3):1-27.

22. Rehman SU, Pupim LB, Shyr $Y$, Hakim R, Ikizler TA. Intradialytic serial vascular access flow measurements. Am J Kidney Dis. 1999;34:471-7.

Este artículo se distribuye bajo una Licencia Creative Commons Atribución-NoComercial 4.0 Internacional. https://creativecommons.org/licenses/by-nc/4.0/

\section{Open Access (c) (7)}

